\title{
Une extension de la notion de sous-pression: Répartition des forces appliquées dans le corps d'une conduite en matériaux poreux sous l'effet d'une pression intérieure
}

\section{An addition to the concept of uplift pressure: Distribution of the forces exerted in the body of a porous pipeline under the influence of internal pressures}

\author{
M. Bouvard, J.L. Kueny, J.L. Thony \\ Institut de Mécanique de Grenoble
}

1. Considération préliminaires : généralisation de la notion de "sous-pression"

\subsection{Les sous-pressions dans les barrages :}

On sait que, initialement au moins, on avait négligé une catégorie de forces - qui s'est avérée par la suite fondamentale - dans le calcul des ouvrages en béton, au contact de l'eau, et spécialement des barrages : les sous-pressions, et c'est le mérite de l'ingénieur français Maurice Lévy de les avoir conçues et introduites dans les calculs.

Le concept était le suivant : dans un barrage type poids, on admettait que l'eau pouvait pénétrer par les fissures du béton, supposées horizontales. D'où une poussée hydrostatique verticale qui se déduisait du poids du barrage. On admettait en général une répartition triangulaire de cette poussée, donc décroissante de l'amont vers l'aval (où on la supposait nulle).

Mais cette décroissance ne pouvait se justifier que s'il $\mathrm{y}$ avait écoulement : la pression ne pourrait que rester constante dans un milieu où l'eau serait immobile.

Certains auteurs [1] ont alors tenté d'extrapoler la notion de sous-pression, initialement liée à l'existence d'une fissure, au cas des matériaux pulvérulents en général, même non fissurés, en la rattachant à l'écoulement poreux qui de toute façon prend naissance au contact entre l'eau et un milieu pulvérulent.

L'expérience de l'éprouvette de "Föppl" [2], citée dans [1], semble bien montrer que cette extrapolation est valable (Fig. 1).

C'est le point de vue que nous allons développer cidessous.

\subsection{Pressions en surface et pressions interstitielles :}

$\mathrm{Si}$ on considère un milieu poreux granulaire saturé d'eau circulant sous l'effet de l'inégalité de la réparti-

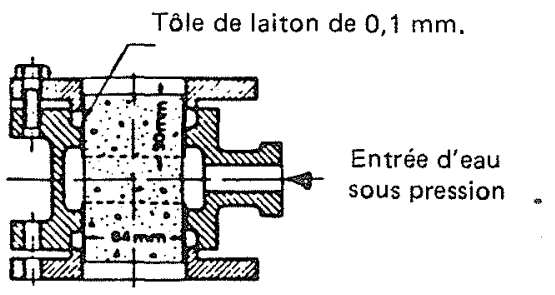

Figure 1. - Montage de Föppl. L'éprouvette soumise à I'essai schématisé ci-dessus casse suivant un plan normal aux génératrices.

tion des pressions, on est conđuit à considérer que le milieu dans son ensemble est soumis à un champ de forces résultant de ces mêmes différences de pressions. Celles-ci sont reliées au champ de vitesses de circulation et à la répartition des perméabilités (caractéristiques propre du terrain), suivant la loi de Darcy:

$$
v=-k i
$$

$v:$ vitesse fictive en un point, définie en admettant que le fluide coule sur la section totale d'un élément de surface.

$k$ : coefficient de perméabilité, constant ou variable, suivant le point et la direction considérée.

$i$ : perte de charge linéaire, comptée sur une ligne de courant de l'écoulement moyen. Si $d s$ représente la longueur élémentaire suivant cette ligne de courant, on a ainsi :

$$
i=\frac{d(p / \omega+z)}{d s}
$$

$\bar{\omega}$ : poids volumique du fluide.

Dans ce cadre, on est conduit à admettre sans difficulté que tout se passe "comme si", à son tour, le milieu fluide exerçait sur les grains solides constituant le maté- 
riau poreux une pression $p$, sur toute la surface des grains, ceux-ci n'étant plus en contact que sur des zones ponctuelles, permettant certes aux contraintes mécaniques de se manifester, mais qui seraient d'importance négligeable par rapport à une surface géométrique quelconque, située dans le milieu.

La notion de sous-pression se définit alors de façon très simple : c'est la pression qu'exerce le fluide en écoulement dans la structure poreuse, avec laquelle il est en contact.

\subsection{Conclusion :}

Le concept de "sous-pression" généralisée conduit à des conclusions fort importantes :

a) La pression statique amont se transformerait en perte de charge de l'écoulement dans le milieu considéré.

Tout se passe comme si la pression statique "pénétrait" en quelque sorte le milieu, pour se transformer en une véritable "force de volume", répartie entre le point amont de la ligne de courant par laquelle le fluide entre en contact avec la structure, et le point aval où il retrouve la pression nulle.

b) En cas de perméabilité variable, la pression amont se concentrerait sur les zones où $k$ serait le plus faible, ce qui pourrait faire apparaître un cas de charge très particulier, éventuellement très défavorable, beaucoup plus que celui considéré habituellement, de pression appliquée à l'amont des surfaces de contact fluide - structure poreuse.

c) Lè dernier aspect soulève une difficulté de principe importante : la répartition de perméabilité dans une structure poreuse est pratiquement impossible à déterminer, et d'autant plus qu'elle est plus faible.

d) Le schéma ainsi développé semble présenter une portée assez ou très générale. Admis depuis longtemps dans les sols pulvérulents, [1] et [2] ci-dessus l'ont étendu au béton. Repris dans [3], [4], [5] et [6] pour être appliqué à du rocher, il semble avoir expliqué plusieurs phénomènes importants.

Nous allons l'appliquer à une structure un peu particulière, celle d'une conduite en éléments de béton, assemblés par des manchons, ces éléments étant préfabriqués.

\section{Exemple d'une conduite en éléments assem- blés par des manchons :}

La conduite se présente alors sous la forme de viroles indépendantes, insérées dans des manchons qui les relient, l'étanchéité étant obtenue par un joint spécial, la plupart du temps en caoutchouc néoprène. La pose est alors relativement simple, dans son principe en tout cas.

Le schéma de la conduite est donné sur la figure 2.

\subsection{Calcul classique (pression supposée appliquée en surface):}

Le calcul des efforts, au cas où la pression est considérée comme appliquée en surface, est simple :

a) Dans la partie courante des viroles, et notamment au milieu, en tout cas loin des extrémités, la paroi est

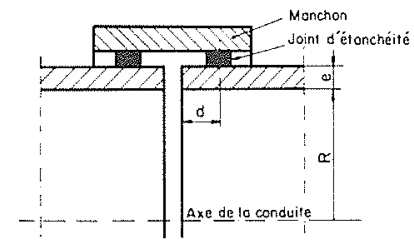

Figure 2

soumise à une force d'extension égale à $p R$, $p$ représentant la pression intérieure, $R$ le rayon intérieur.

Si on admet que la contrainte mécanique résultante $n$ est uniforme (ce qui, mécaniquement, ne serait valable que pour des tuyaux de faible épaisseur), elle correspond à la formule très simple et bien connue :

$$
n=p \frac{R}{e}
$$

si $e$ représente l'épaisseur de la virole.

b) Au droit du joint, lorsque la virole pénètre dans le manchon, l'effort d'extension de la partie courante s'étale sur une certaine longueur, et la contrainte résultante est plus faible.

Les conditions de résistance de la paroi de la viroie, au voisinage du manchon, sont donc meilleures, suivant les critères d'appréciation ci-dessus, que dans leur partie courante.

\subsection{Calcul avéc "pénétration" de la pression:}

En réalité, des incidents survenus dans des conduites en matériaux poreux laissent penser que, contrairement à ce qu'on pourrait croire, ce calcul est trop optimiste.

Aussi, nous allons appliquer les considérations développées ci-dessus, quant à la "pénétration" du milieu par la pression.

Plusieurs zones sont à considérer :

a) La partie courante des viroles, loin de ses extrémités (notamment, sur l'axe de celles-ci)

Perméabilité homogène du matériau : Le tracé de l'écoulement est alors très simple. Sauf si l'épaisseur de la paroi était vraiment très grande, la répartition de la perte de charge de l'écoulement sur toute la paroi équivaudrait à une poussée élémentaire $d P / d R$, chaque conduite élémentaire, d'épaisseur $d R$, recevrait donc une pression constante, rapportée à $d R$. La virole serait constituée en quelque sorte par une infinité de tubes d'épaisseur $d R$, recevant chacun une pression $d P$, travaillant pratiquement de la même façon que si la pression était appliquée à la surface intérieure.

Perméabilité du matériau variable sur l'épaisseur de la conduite: Deux cas peuvent à priori se présenter : - le matériau est moins perméable à l'intérieur de la paroi. Ceci pourrait se produire, par exemple, si le matériau (béton) se trouvait de meilleure qualité à l'intérieur, pour des raisons liées au processus de fabrication (centrifugation par exemple); ce cas se ramènerait encore plus à celui de la conduite chargée en surface, exactement même, si on supposait que, sur la surface intérieure, la 
virole avait reçu un enduit étanche (ce qui représente l'extrême de ce qui peut arriver en ce qui concerne la différenciation des perméabilités). Simplement, les "conduites élémentaires", évoquées ci-dessus seraient alors soumises à un effort de compression radiale, correspondant au fait que les conduites élémentaires les plus près du centre appuieraient sur celles qui sont à la paroí, et ainsi toutes contribueraient à la résistance à la pression intérieure.

- le matériau est moins perméable à l'extérieur. Le cas de charge est alors tout différent. Si on suppose -à l'extrême - que la couche extérieure a reçu un enduit étanche (de perméabilité très faible ou nulle à la limite), l'écoulement poreux induit par la pression intérieure provoquera une perte de charge élevée, mais voisine de la paroi extérieure. Celle-ci solliciterait à son tour les autres conduites élémentaires, plus centrales, par traction radiale, ceci à condition que le matériau qui constitue la conduite résiste bien à cet effort de traction, ce qui suivant les matériaux en cause n'est pas toujours le cas.

En réalité, on ne sait pas grand chose à priori de la répartition réelle des perméabilités dans l'épaisseur de la conduite, sinon ceci :

- une variation de perméabilité de 1 à 10 survient très facilement dans le cas d'un matériau tel que le béton. Il suffit que celui-ci soit ou enrichi ou appauvri localement en matériau fin (sable ou même ciment) pour que la. perméabilité diminue ou augmente par rapport à la moyenne:

- à ces variations de perméabilité seront alors associées des variations de pertes de charge de l'écoulement dans la paroi, susceptibles de conduire à des répartitions totalement inconnues de la pression intérieure, et à un système de contraintes mécaniques impossible à préciser.

\section{b) Extrémités des viroles:}

Evidemment, un phénomène analogue se produira au droit des joints (en caoutchouc ou en matière plastique) placés entre chaque virole et le manchon qui les réunit, mais le cas de charge sera beaucoup plus complexe.

La pression intérieure à la conduite, transformée en perte de charge de l'écoulement dans la paroi, se traduira par une force appliquée répartie sur une longueur très limitée correspondant à l'épaisseur du joint. Comme cette longueur n'est en général pas très grande, le "gradient" de pression ainsi appliqué pourra s'avérer très important. Il serait aussi associé, comme le montre le calcul présenté dans la suite, à des effets d'extrémité très importants (concentration de la charge sur une portion très faible de l'épaisseur de la conduite).

De toute façon, le cas de charge résultant n'aura rien à voir avec celui qu'on obtiendrait en considérant que la pression est appliquée à la surface des viroles : c'est bien alors la partie courante de la conduite qui est la plus chargée.

\subsection{Essais d'eau et écoulement poreux :}

Souvent, les viroles, dont il est question ici, sont essayées à une certaine pression intérieure, supérieure, bien sûr, à la pression de service pour contrôler leur bonne tenue.

Plusieurs cas peuvent alors se présenter :

a) Avant l'essai, les viroles étaient stockées à sec, et l'essai est rapide (de l'ordre de la minute).

Immédiatement au début de l'essai, tout se passe effectivement comme si la conduite était mise en pression en surface, puisque l'eau n'a pas encore pu pénétrer le matériau et "transférer" la pression à l'intérieur de la paroi. Si on veut interpréter cet aspect dans le cas général, on peut dire que la perte de charge de l'écoulement poreux est concentrée à la surface intérieure des viroles, puisque l'eau n'a pas encore eu le temps d'y entrer très profondément.

A mesure que l'essai se prolongerait, au contraire, le cas de charge évoluerait, pour tendre vers le cas où, l'écoulement de régime permanent à travers la paroi étant atteint, la pression se répartirait en réalité sur toute l'épaisseur de la conduite, comme indiqué cidessus.

Tout dépendrait alors du rapport entre la durée de l'essai $t_{e}$, et le temps nécessaire à l'établissement du régime permanent d'écoulement à travers la paroi, soit $t_{r p}$. Mais si :

$$
t_{e}<<t_{r p}
$$

la virole sera essayée suivant un cas de charge qui n'aurait pas de rapport avec celui existant après une durée d'imbibition d'importance notable, de l'ordre de $t_{r p}$, et la résistance de la conduite ne pourra être que surestimée.

Des considérations très simples permettent d'avoir une idée de $t_{r p}$, pour la partie courante des viroles.

Admettons que l'épaisseur de la paroi soit faible devant leur rayon, ce qui permet d'écrire que la vitesse $u$ de l'écoulement poreux est constante et indépendante du rayon. On a alors:

$$
u=\frac{k H}{\rho-R}
$$

Si $\rho$ représente le rayon atteint par l'écoulement, $R$ le rayon intérieur de la conduite et $H$, la charge hydraulique appliquée lors de l'essai.

La fuite correspondante sert à faire progresser de $d \rho$ la zone atteinte par l'écoulement avec, si $m$ est le coefficient de porosité du matériau :

$$
m \frac{d \rho}{d t}=\frac{k H}{\rho-R}
$$

d'où on déduit immédiatement, après intégration :

$$
t=\frac{m e^{2}}{2 k H}
$$

si, par exemple,

$$
k=10^{-8} \mathrm{~m} / \mathrm{sec} ., e=0,10 \mathrm{~m}, H=100 \mathrm{~m}, \mathrm{~m}=0,10 \text {, }
$$

on trouve $t=500$ secondes, soit un peu moins de 10 minutes. 


\section{b) Avant l'essai, les viroles sont stockées dans l'eau}

L'essai est alors représentatif du cas de charge réel, en ce qui concerne la partie courante de la virole en tout cas : l'écoulement permanent à travers la paroi s'établit dès que l'eau est mise en pression, du moins si la paroi est parfaitement humide lors de la montée en pression.

\section{c) Les effets d'extrémité}

Comme on l'a vu, la portion de la virole voisine du manchon est soumise à un cas de charge très particulier, et le système d'essai devrait en reproduire les caractéristiques:

- sous l'aspect du régime permanent de l'écoulement; - sous l'aspect des conditions aux limites, concernant la manière dont la virole est emmanchée, avec la pièce d'étanchéité.

\subsection{Conclusion :}

De toute façon les conditions dans lesquelles travaille une conduite constituée de matériaux poreux sont difficiles à analyser :

- soit par le calcul : en particulier, l'hypothèse suivant laquelle la pression est appliquée en surface ne correspond pas au cas de charge;

- soit par un essai, s'il ne dure pas assez longtemps (quand les viroles sont stockées "à sec"), ou s'il ne reproduit pas les effets d'extrémités, notamment l'intervention du joint dans la répartition des pressions.

Dans le cas le plus extrême, l'erreur ainsi introduite dans l'appréciation de la résistance de la pièce est inconnue, dans la mesure notamment où la répartition des perméabilités du matériau ne serait pas trop variable ou aléatoire.

\section{Calcul hydraulique :}

Il est néanmoins possible d'avoir - au titre d'une première étape- une idée des problèmes ainsi soulevés, dans le cas bien entendu où on connaîtrait la perméabilité du matériau. Restera alors l'influence des variations de celle-ci, sous la dépendance des méthodes d'exécution. L'appréciation de cet aspect dépendrait de données sur la répartition des perméabilités. Aussi, faute de données concernant ce point, nous nous limiterons à la première étape.

\subsection{Principe de la solution :}

L'écoulement dans la paroi est a priori de révolution. Cependant, en nous limitant au cas ou $R / e$ est suffisamment grand, on considérera que l'écoulement à travers la paroi est plan (bidimensionnel).

L'erreur ainsi introduite est certainement négligeable, en tout cas devant les inconnues correspondant à d'autres aspects du problème (du ressort, par exemple, de la seconde étape).

A partir du moment où on admet l'homogénéité des perméabilités, rien n'empêche d'introduire une anisotropie de celle-ci, qui pourrait correspondre à une "anisotropie" de fabrication. Par exemple, si les tuyaux sont centrifugés, on conçoit que le serrage "cylindrique" du matériau soit plus puissant que le serrage "axial", ce qui pourrait correspondre à une réduction de la perméabilité radiale par rapport à la perméabilité axiale.

Autrement, il suffit d'écrire que l'écoulement supposé plan comme indiqué ci-dessus, satisfait l'équation de Laplace, c'est-à-dire que :

$$
\frac{\partial^{2} h}{\partial x^{12}}+\frac{\partial^{2} h}{\partial y^{2}}=0
$$

après avoir posé :

$$
x^{i}=\left(\frac{K y}{K x}\right)^{1 / 2} x
$$

$K x$ et $K y$ représentant les coefficients de perméabilité suivant les axes $O x$ et $O y$. Ceci avec :

$$
h=\frac{p}{\bar{\omega}}+z
$$

En réalité, compte tenu des pressions mises en jeu, le terme $z$ peut être négligé.

\subsection{Pratique du calcul :}

Les techniques d'intégration de ce type d'équation sont maintenant classiques. C'est une méthode aux différences finies qui a été choisie. Le maillage en $x$ et $y$ est à pas constants dans la direction transversale et à pas variables suivant l'axe de la conduite. Sous le joint et dans la partie amont où la charge hydraulique évolue rapidement, les mailles sont approximativement carrées. Dans la partie semi-infinie, on utilise une progression géométrique du pas des mailles, arrêté sur une frontière à distance finie. On impose alors un gradient longitudinal de charge nul, et on vérifie après intégration que la répartition transversale obtenue pour les pressions est bien linéaire. Si ce n'est pas le cas, on éloigne la frontière.

La taille du maillage est choisie pour garantir la précision de la discrétisation : $30 \times 100$ mailles sont ici nécessaires.

La charge hydraulique est imposée sur les surfaces intérieures et extérieures de la conduite. Sous le joint, on utilise un gradient normal de charge nul.

L'équation de Laplace a été discrétisée avec un opérateur précis au deuxième ordre à l'in térieur du domaine, ainsi qu'aux frontières, avec conditions aux limites du type Von Neuman.

La résolution du système s'effectue par la méthode des directions alternées et les isovaleurs de la pression sont obtenues avec un programme de tracés automatiques.

\subsection{Contrôle des résultats par des essais dans une cuve à analogie électrique :}

Les résultats de la simulation numérique de l'équation de Laplace qui s'applique au cas considéré peuvent être comparés au résultat d'une simulation physique au moyen de l'analogie électrique. 
Cette technique, d'usage très commode, permet d'obtenir sans difficulté les lignes équipotentielles du réseau hydrodynamique à l'intérieur de la paroi de la conduite et en particulier près du joint.

Moyennant l'assimilation légitime de la charge à la pression, les lignes équipotentielles correspondent aux lignes isobares. L'anisotropie peut également être simulée, en reproduisant la même distorsion que celle introduite dans l'équation de Laplace.

Les résultats des essais en analogie électrique, effectués sur l'installation des travaux pratiques de l'ENS d'hydraulique de Grenoble, recoupent parfaitement les solutions obtenues par la voie numérique.

\subsection{Résultats :}

\section{a) Partie courante de la virole}

On ne trouve rien de bien particulier, sinon que l'homogénéité de la répartition des perméabilités entraîne l'homogénéité de la répartition des charges : les équipotentielles sont espacées régulièrement sur toute l'épaisseur de la conduite.

Reste la question de l'homogénéité des perméabilités, du ressort d'un autre univers que celui du calcul.

\section{b) Extrémité de la virole}

Les caractéristiques du joint ont été paramétrées par deux valeurs, rapportées à l'épaisseur $e$ de la conduite : - son épaisseur, prise égale à $e / 3$ et $2 e / 3$;

- la distance de l'axe du joint à l'extrémité de la virole, prise égale à $0,5 e ; 5 e / 6 ; 5 e / 3$.

Nous avons considéré une différence de pression $\Delta p / \bar{\omega}$ unitaire entre l'intérieur et l'extérieur de la virole, la répartition des pressions dans un cas réel étant strictement proportionnelle à la répartition obtenue pour $\Delta p / \bar{\omega}=1$.

Les figures $3 a, 3 b, 3 c$, représentent l'essentiel du résultat obtenu par le calcul ou dans la cuve à analogie électrique pour $K x / K y=10$.

On notera :

- que la répartition des pressions interstitielles à l'aval du joint (donc à droite de celui-ci sur la figure 4) corres. pond à une forte concentration des pressions, quand le joint est mince. Cette surcharge doit alors être absorbée : soit par l'effet de courbure du cylindre, soit par traction

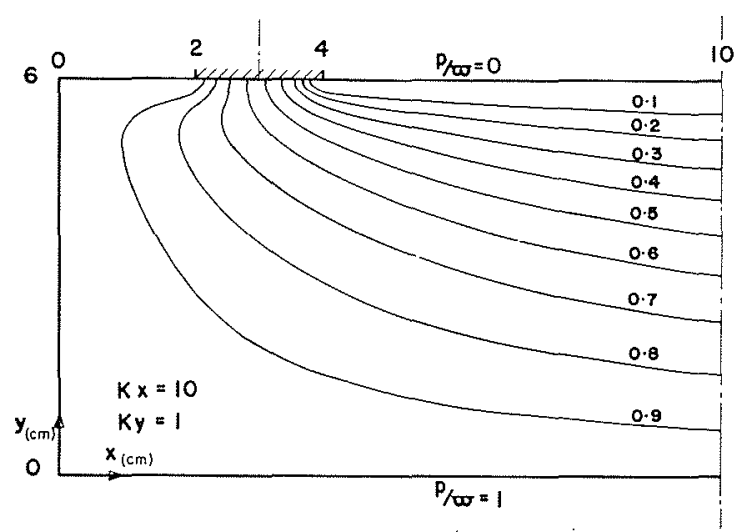

10

Figure $3 a$

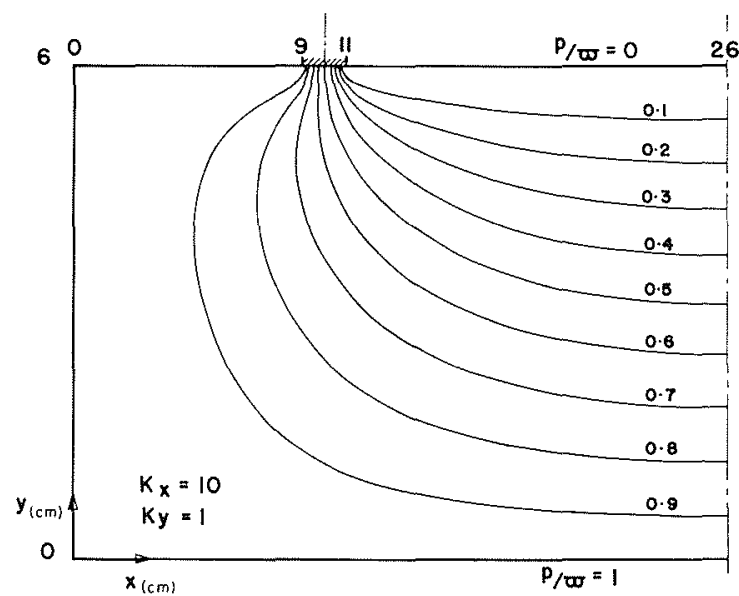

Figure $3 b$

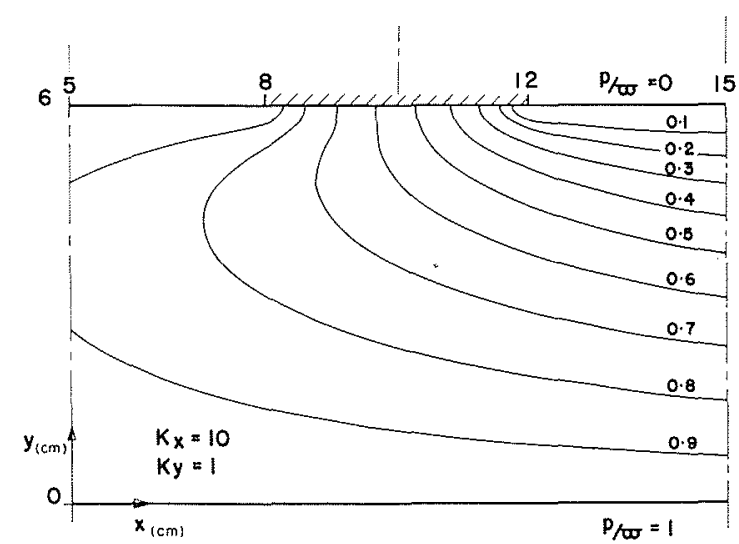

Figure 3c

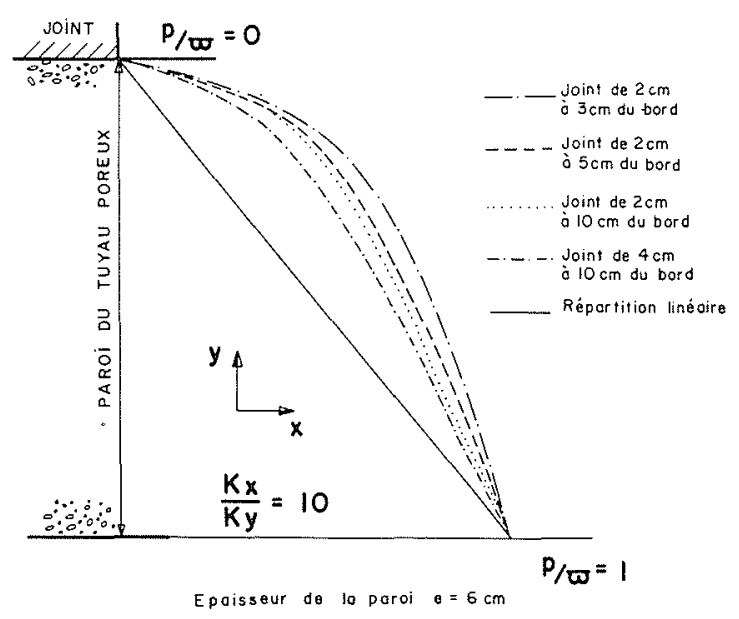

Figure 4

du matériau, qui se manifesterait plus tôt que l'effet de courbure, puisqu'il met en jeu une déformation bien moindre;

- que le résultat, dans cette zone est relativement indépendant de la distance du joint aux extrémités de la virole. 


\section{Conclusion - Calcul des efforts}

Les résultats obtenus ci-dessus portent simplement sur la répartition de la pression intérieure, à l'intérieur de la structure, du fait de la pénétration par l'écoulement poreux consécutif à la mise en pression de la conduite.

On notera combien ils peuvent être différents de ceux correspondant à une mise en pression à la paroi jusqu'à, même, n'avoir aucun rapport avec les résultats correspondants à cette hypothèse. La répartition des pressions le long du joint, la concentration des pressions à l'aval de celui-ci, constituent des phénomènes dont l'analyse n'est possible que dans le cadre considéré ci-dessus. Lui seul permettrait d'expliquer la rupture de certaines viroles en service, pourtant largement dimensionnées et essayées.

Resterait une étape ultérieure, qui consisterait à étudier les contraintes dans la structure à partir des efforts ainsi mis en évidence. Le calcul sera alors beaucoup plus complexe. On devrait bien entendu tenir compte de la courbure de la pièce, en introduisant le fait que le cas de charge, les contraintes, restent de révolution.

Evidemment, un tel calcul ne peut ètre conduit que par la méthode des éléments finis, les considérations développées ci-desssus se bornant à définir les charges appliquées, point par point.

Mais on peut d'ores et déjà être certain que le résultat n'aura qu'un rapport très lointain avec celui que donnerait l'analyse classique, avec pression appliquée à la paroi intérieure, supposée entièrement étanche.

\section{Références bibliographiques}

[1] SERAFIN L. - A subpressão nas barragens. Thèse, L.N.E.C., Lisbonne.

[2] FOPPL. - Mitteilungen aus dem Mecanisch - Technischen Laboratorium (Munich, Tome 27, 1900).

[3] BOUVARD M., PINTO N. - Aménagement Capivari-Cachoeiva - Etude du puits en charge. "La Houille Blanche" $n$ " 7, 1969.

[4] BOUVARD M., PINTO N. - Les ouvrages en charge de l'aménagement Capivari-Cachoeiva. Conclusions sur quelques aspects généraux des souterrains à forte pression. "La Houille Blanche" $n^{\circ} 4,1971$.

[5] BOUVARD M. - Les fuites des galeries en charge. Rôle du revêtement, des injections, du terrain. "La Houille Blanche" $\mathrm{n}^{\circ} 4,1975$.

[6] BOUVARD M., NIQUET J. - Ecoulements transitoires dans les massifs autour d'une galerie en charge. "La Houille Blan" che" n' 3, 1980. 